\title{
Capacity Evaluation for CDMA Cellular Systems
}

\author{
Gulshan Karmani and Kumar N. Sivarajan
}

\begin{abstract}
In this paper, we find bounds and approximations for the capacity of mobile cellular communication networks based on Code Division Multiple Access (CDMA). We develop efficient analytic techniques for capacity calculations of CDMA cellular networks. Each cell is modeled as an independent $M / G / \infty$ queue and traffic capacity assessed based on the maximum Erlang traffic that leads to acceptable link quality with high probability. Subsequently, approximations and bounds for the outage probability and hence traffic capacity are obtained using Asymptotic Expansions and Large Deviations Theory. Numerical examples, considering uniform and Normalized Truncated Gaussian user density in the system are evaluated. The propagation model we consider takes care of distance and lognormal shadowing losses.
\end{abstract}

\section{INTRODUCTION}

$\mathrm{T}$ HE development of the Code Division Multiple Access (CDMA) scheme is mainly for capacity reasons. CDMA systems are claimed to have higher capacity than FDMA/TDMA systems. Capacity is a very important parameter for the network operator of any system. However capacity in CDMA systems is not as easy to evaluate as in TDMA/FDMA based systems. This is because capacity is limited by the interference from all other users in CDMA based systems. This paper contributes to the development of efficient techniques to assess the capacity of CDMA based systems more accurately.

We begin in section II with a review of some existing capacity analyses, their shortcomings and our proposed work. In Section III we discuss the models and definitions for the work in later sections.

In Section IV we examine methods for quantifying the interference from other cells and find approximations for the outage probability. A large portion of this section is based on Edgeworth Asymptotic Expansions which are used to reduce the error in the Central limit Theorem (CLT) approximations, when the latter are applied to a sum of small number of random variables.

In Section $\mathrm{V}$ we determine upper bounds for the outage probability using Large Deviations theory. An efficient way to calculate the exact Chernoff bound without finding the distribution of the interference random variables is given, which can be easily extended to the case of nonuniform user densities in the system.

In Section VI lognormal shadowing is included into the propagation model and in this case only the CLT approximation with Edgeworth corrections is used to estimate the outage probability. We conclude our work in Section VII highlighting the scope for future work in this area.

\section{Existing CAPACITY ANALYSES}

The paper by Gilhousen et.al [1] is the earliest that considers the capacity of multi-cellular CDMA with emphasis on the analysis of interference from other cells. It is assumed that there is an

This work was carried out when the authors were with the the Indian Institute of Science, Bangalore. Gulshan Karmani (guls@sasken.com) is presently with Sasken Communication Technologies, Bangalore. Kumar N. Sivarajan (kumar@tejasnetworks.com) is with Tejas Networks, Bangalore. equal number of users per cell and they are evenly (and continuously) spread over each cell. Perfect power control to a fixed signal power is assumed and the propagation model accounts for independent lognormal shadowing along with distance losses. With the assumption of power control by the nearest Base Station (BS), they obtain bounds on the first two moments of the random variable (rv) corresponding to interference.

Unlike in [1] (where they take an equal number of users in each cell), in this paper by Viterbi et.al [2] each cell is modeled as an $M / G / \infty$ queue, and hence the number of active calls is a Poisson rv with mean equal to the traffic offered to each cell. Gaussian approximation and chernoff bound are obtained for the outage probability and compared to simulation results.

Another paper by Evans and Everitt [3] is an extension to the work in [2]. They also assume an $M / G / \infty$ model for each cell, obtain the distribution function for the interference rv (considering only distance losses). Later, similar bounds and approximations as in [2] are obtained and compared to simulations.

\section{A. Shortcomings of Existing Capacity Analyses}

1. Bounds and approximations obtained in [2] and [3], show a significant discrepancy from the simulations. The chernoff bound in both the cases is very conservative. On the other hand the Gaussian approximation seems to underestimate the outage probability (the term defined later for CDMA systems) in the tail portion, which means capacity is overestimated.

2. The method of obtaining bounds and approximations for capacity using the distribution function of the rv corresponding to interference [3] is very complicated, besides being less accurate. Moreover the extension of this method to the case of nonuniform user density is very complicated.

\section{B. Proposed work}

1. The main focus of this paper is to obtain theoretical bounds and approximations for the capacity of systems employing CDMA, more accurately. We will determine the approximations and bounds using asymptotic expansions and large deviations theory, considering only distance losses. Later we will extend the results to the cases where lognormal shadowing is taken into account and the mobiles are power controlled by the Base Stations (BSs) having least attenuation.

2. Unlike the approach in [3], we will obtain the bounds and approximations using numerical integrations [7], as this is more accurate and efficient.

\section{Models AND DEFinitions}

\section{A. Soft Blocking and Outage Probability in CDMA}

In CDMA based systems, all users share a common spectral frequency allocation over the time that they are active. Hence, new users can be accepted as long as there are codes available to 
be assigned. We assume that there are a sufficiently high number of codes available such that the probability of a new arrival finding them busy is negligible. Then, blocking in CDMA systems will be defined to occur when the interference level, due primarily to other user activity, reaches a predetermined level above the background noise level. This kind of blocking can be called as soft blocking.

To calculate the outage probability, we must determine the probability that an arbitrary call arriving anywhere in the cellular network receives a reverse link SIR that is insufficient for acceptable Quality of Service (QoS). Since symmetry exists in the hexagonal system we consider calls that connect to a particular BS. Associate with this target BS and its cell the index 1.

Because of the standard power control assumptions, an arriving call would be received at BS 1 with unit signal power. We can thus translate the SIR requirement into a constraint on the total interference at BS 1 at the call arrival time. In summary the expression for outage probability is reduced to,

$$
P_{\text {out }}=\operatorname{Pr}\left(I_{\text {tot }} \geq \Gamma\right)
$$

where $I_{t o t}$ is a rv representing the total interference power received at BS 1 in the network at some time. $\Gamma$ is a measure of the size of the CDMA system (Capacity) and is related to the system bandwidth $(W \mathrm{~Hz})$, data rate $(R \mathrm{bps})$, and the required bit energy to interference density ratio $\left(E_{b} / I_{0}\right)$ by [1]

$$
\Gamma=\frac{W / R}{E_{b} / I_{0}} .
$$

\section{B. The $M / G / \infty$ Traffic Model}

A fairly well known model for CDMA based cellular systems is the $M / G / \infty$ model for each cell. This model is justified in [3]. The model implies that each cell of our network behaves like an independent $M / G / \infty$ queue. Let the mean time between call arrivals be $1 / \lambda$ seconds and the mean call holding time be $1 / \mu$ seconds. Let $N$ be the rv representing the number of active calls in the cell in steady state. Then $N$ has a Poisson distribution [4]. If $A=\lambda / \mu$

$$
\operatorname{Pr}(N=n)=e^{-A} \frac{A^{n}}{n !}
$$

Hence this model allows us to replace the deterministic number of users in each cell by an independent Poisson rv for each cell.

\section{Propagation Model}

The mobile radio environment is generally modeled as a timevarying fading channel, where the attenuation incurred by the transmitted signal is given by,

$$
\alpha(d) \propto r^{-\gamma} \cdot G^{2} \cdot R^{2}
$$

where, 1. $r^{-\gamma}$ is the path loss with path loss exponent $\gamma$ (PLE), and $r$ is the distance between the transmitter and the receiver.

2. $G^{2}$ is the shadowing $r v$. This is due to variations in terrain contour and to shadowing from buildings. $G^{2}$ is lognormally distributed, i.e.,

$$
G^{2}=10^{-\xi^{\prime} / 10}
$$

where

$$
\xi^{\prime} \sim N\left(\mu, \sigma^{2}\right)
$$

Typical value for $\sigma$ is $8 \mathrm{~dB}$ [10] and the model makes sense for $\mu=3 \sigma$.

3. $R^{2}$ is the Rayleigh fading component due to multipath. The density function of $R$ is given as, for $x \geq 0$,

$$
f_{R}(x)=\frac{2 x}{E\left(R^{2}\right)} \exp \left(\frac{-x^{2}}{E\left(R^{2}\right)}\right) \text {. }
$$

\section{CLT CORRECTION FOR THE OUtAGE PRobability APPROXIMATIONS}

In this section, we discuss outage probability calculations and obtain approximations for the outage probability. We first characterize the interference rv from other users. We then find approximations to the outage probability by Central Limit Theorem (CLT) and then apply a correction to the CLT approximation using asymptotic expansions.

\section{A. Characterization of Interference}

We know that the capacity of CDMA cellular systems depends on the interference received from all other users in the system. The outage probability (1) that determines the capacity, solely depends on the total interference received at a Base Station (BS) from all users other than the desired one. This total interference is the sum of the interference from each active user and is a rv due to the random positions of users in the system.

\section{B. Individual Interference Random Variable}

Since the total interference rv $I_{t o t}$ (defined later) is a sum of the interference from each active user in the system, we will be calling the interference from each user as the individual interference $\operatorname{rv} I_{k}$ (defined later). It is necessary to characterize the individual interference rv to approximate the outage probability and hence the capacity of CDMA cellular systems.

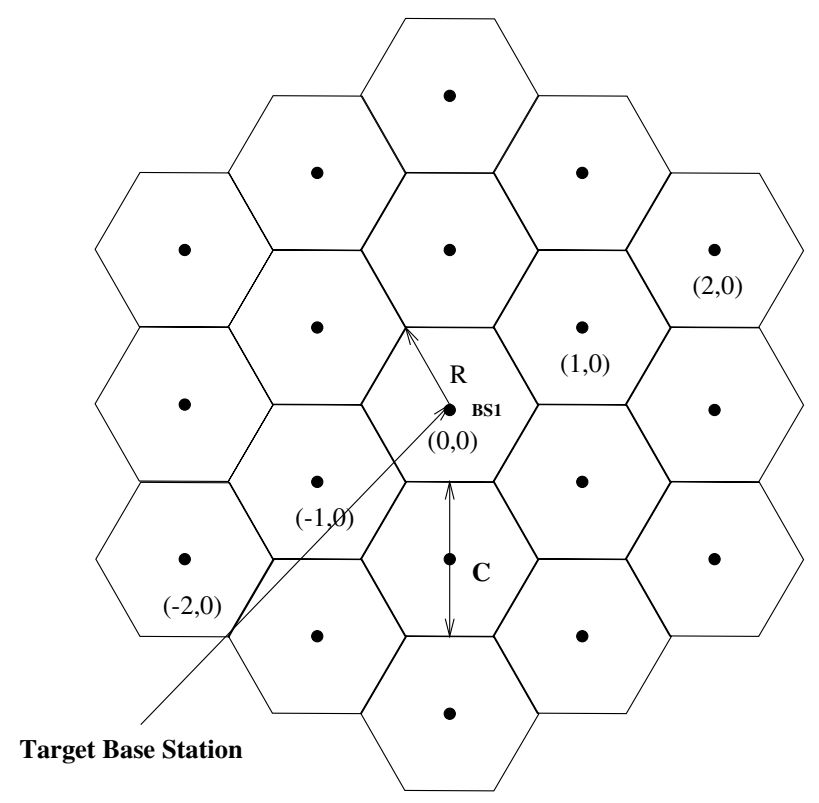

Fig. 1. 2-Dimensional Hexagonal Tiling 
To characterize the individual interference rv, we make the following assumptions:

1. A uniform hexagonal cellular layout as in Fig. 1, where we have normalized the radius of each cell to $R=\frac{1}{\sqrt{3}}$. This corresponds to inter-cell distance $C$ of unity.

2. The propagation model of (3) and we consider only the distance losses in this section and in section V. Lognormal shadowing is considered in section VI. The inefficiency in power control and Rayleigh fading is averaged out and its effects are encapsulated in the $E_{b} / I_{0}$ requirement.

3. Density of users is taken to be uniform initially and later we consider a particular case of non-uniform user density in the system.

4. Due to the symmetrical hexagonal layout, $P_{\text {out }}$ will be same for the MS everywhere in the system. So we may consider only the active calls that are connected to a particular BS for $P_{\text {out }}$ calculation, and associate with this target BS and its cell the index 1. Thus we label the target BS as BS 1 .

5. Because of the standard power control assumptions, mobiles are received at their own BSs at unit signal power.

6. Target cell and the first two tiers of cells alone contribute significantly to the total interference at the target BS 1 .

7. An equal traffic of $A$ Erlangs is offered to each cell in the system.

Let there be $M$ cells in the network. We consider a reference cell 1 . The interference caused to the transmissions of cell 1 is due to the transmissions by the users of the same cell and those of cells with indices $2, \ldots, M$. We assume that the interference rvs from mobiles in an arbitrary cell $i$ are independent and identically distributed (iid) with cumulative distribution function (CDF) $F_{i}$, and the interference rvs from different cells are independent. Given $N_{i}$ calls in cell $i$ the total interference received at $\mathrm{BS} 1$ is given by

$$
I_{t o t}=\sum_{i=1}^{M} \sum_{j=1}^{N_{i}} I_{i j}
$$

where $I_{i j}$ is the interference from the $j$ th mobile in cell $i$. Due to the $M / G / \infty$ assumption described in Section III-B, and equal traffic offered to each cell in the system, $N_{i}$ is a Poisson rv with mean A. It can be shown that,

$$
I_{t o t} \doteq \sum_{k=1}^{N} I_{k}
$$

where $\doteq$ denotes equality in distribution. $N$ is a Poisson rv with parameter $M \cdot A$ and $I_{k}$ is a individual interference rv with CDF $M^{-1} \sum_{i=1}^{M} F_{i}$ [3]. For convenience we will denote the individual interference rv $I_{k}$ as $I$ (whenever referred without the summation). In (4), the interfering cells are combined and the total traffic in the system is considered.

\section{B.1 Moments of Individual Interference Random Variables}

Taking into account all the assumptions listed above, we can calculate the $n^{t h}$ moment of the individual interference rv $I$ (which is a function of the interfering mobile's location $(x, y)$ ), using:

$$
E\left(I^{n}\right)=\iint_{\mathcal{R}} I(x, y)^{n} f(x, y) d x d y
$$

where $\mathcal{R}$ is the 2-D Hexagonal Tiling consisting of two tiers shown in Fig. 1.

$$
I(x, y)=\left(\frac{r_{i}(x, y)}{r_{1}(x, y)}\right)^{\gamma}
$$

$f(x, y)=$ User Density $=\frac{1}{M(\text { Area of each hexagon })}$

$r_{i}(x, y)=$ Distance of the interfering mobile in $\left(i^{t h}\right)$ cell from its own BS.

$r_{1}(x, y)=$ Distance of the interfering mobile in $\left(i^{t h}\right)$ cell from the Target BS 1 .

$\gamma=$ path loss exponent (PLE).

\section{B.2 Outage Probability Expression}

Using (4) and (1) we arrive at a simple expression for the outage probability in the network which is given by the probability of a Compound Poisson Sum [5] exceeding the threshold (system size $\Gamma)$ :

$$
P_{\text {out }}=\operatorname{Pr}\left(\sum_{k=1}^{N} I_{k} \geq \Gamma\right)
$$

\section{Gaussian Approximation of Compound Poisson}

Since $N$ has a Poisson distribution the outage probability $P_{\text {out }}$ is given by,

$$
P_{\text {out }}=\sum_{n=0}^{\infty} \operatorname{Pr}\left(\sum_{k=1}^{n} I_{k} \geq \Gamma \mid N=n\right) p(n)
$$

Since $N$ is Poisson rv with mean $M A$ (overall load offered to the system), We get,

$$
P_{\text {out }}=\sum_{n=0}^{\infty} \operatorname{Pr}\left(\sum_{k=1}^{n} I_{k} \geq \Gamma \mid N=n\right)\left(\frac{e^{-M A}(M A)^{n}}{n !}\right)
$$

Consider (7). Assuming that the summation involves a large number of rvs, we can apply the Central Limit Theorem (CLT) to the sum

$$
S_{n}=\sum_{j=1}^{n} I_{j}
$$

Let the mean and variance of $S_{n}$ be,

$$
\begin{gathered}
\mu_{n}=E\left(S_{n}\right)=n E(I), \\
\sigma_{n}^{2}=\operatorname{var}\left(S_{n}\right)=n \operatorname{var}(I) .
\end{gathered}
$$

where $I$ is the individual interference rv in the summation $S_{n}$. Then the approximate outage probability for

$$
Q(x)=\frac{1}{\sqrt{2 \pi}} \int_{x}^{\infty} e^{-t^{2} / 2} d t
$$

is

$$
P_{\text {out }} \approx \sum_{n=0}^{\infty} Q\left(\frac{\Gamma-\mu_{n}}{\sigma_{n}}\right)\left(\frac{e^{-M A}(M A)^{n}}{n !}\right)
$$




\section{C.1 Results}

Using a sufficiently large number of terms around the mean in (8) we obtained $P_{\text {out }}$. We have compared the results so obtained with the simulation results in Fig. 2 and Fig. 3. We consider outage probability in the range $10^{-4}$ to $10^{-1}$. The PLE used is 4 , being a typical value. $\Gamma$ takes values 100 and 20 . These parameters are chosen to compare our results with the previous work in the literature. In all plots the ordinate represents the base 10 logarithm of the outage probability while the abscissa corresponds to the offered traffic per cell divided by $\Gamma$. Simulation points are shown with confidence intervals of $95 \%$. It is seen that the CLT approximation overestimates the capacity of the system in the tail portion, and this is a matter of concern. We apply an asymptotic expansion in the next section to reduce the error.

\section{C.2 Method of Simulation}

Similar method for simulation as in [3] is used. For a given set of system parameters and an offered traffic value, the simulation point is generated directly using a Monte carlo technique. This involves repeatedly generating a random (Poisson) number of users in the entire hexagonal layout of $M=19$ cells, with mean equal to 19 times the corresponding offered traffic. Each user is assigned a uniform location in the system and its interference is calculated. In each trial, the total interference at the target BS 1 is determined from which the outage condition can be checked. The simulated outage probability is then obtained by taking the ratio of the number of outage events to the total number of trials.

\section{Edgeworth Asymptotic Correction}

The Edgeworth expansion is an asymptotic expansion in terms of derivatives of the normal distribution function. This expansion is useful in removing the error in CLT approximation, making use of the moments higher than the first and second.

The expansion is given in [6] in terms of

$$
\begin{gathered}
Z(x)=\frac{1}{\sqrt{2 \pi}} e^{-x^{2} / 2} \\
P(x)=\frac{1}{\sqrt{2 \pi}} \int_{-\infty}^{x} e^{-t^{2} / 2} d t
\end{gathered}
$$

and

$$
Q(x)=\frac{1}{\sqrt{2 \pi}} \int_{x}^{\infty} e^{-t^{2} / 2} d t
$$

where

$$
P(x)+Q(x)=1
$$

Let $Y_{i}(i=1,2, \ldots, n)$ be $n$ independent rvs with mean $m_{i}$, variance $\sigma_{i}^{2}$, and higher cumulants $\kappa_{r, i}$, where $\kappa_{1, i}=m_{i}$, $\kappa_{2, i}=\sigma_{i}^{2}, \kappa_{3, i}=\mu_{3, i}, \kappa_{4, i}=\mu_{4, i}-3 \mu_{2, i}^{2}, \mu_{2, i}=\sigma_{i}$ is the second central moment and $\mu_{3, i}, \mu_{4, i}$ are third and fourth central moments of the rvs $Y_{i}$. Then an asymptotic expansion with respect to $n$ for the cumulative distribution function of

$$
X=\frac{\sum_{i=1}^{n}\left(Y_{i}-m_{i}\right)}{\left(\sum_{i=1}^{n} \sigma_{i}^{2}\right)^{\frac{1}{2}}}
$$

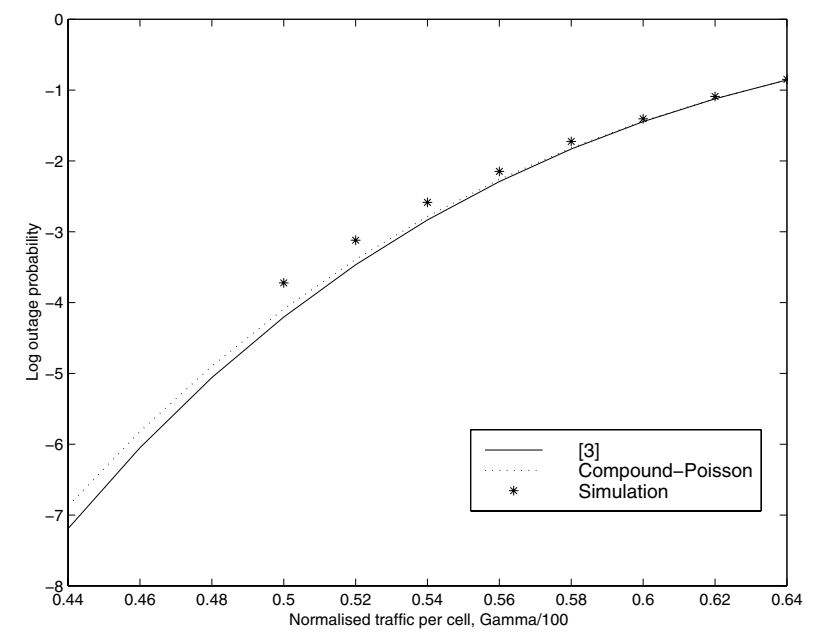

Fig. 2. Comparison of Compound Poisson Method, Approximation in [3] and Simulation for $\Gamma=100$. The user density is uniform.

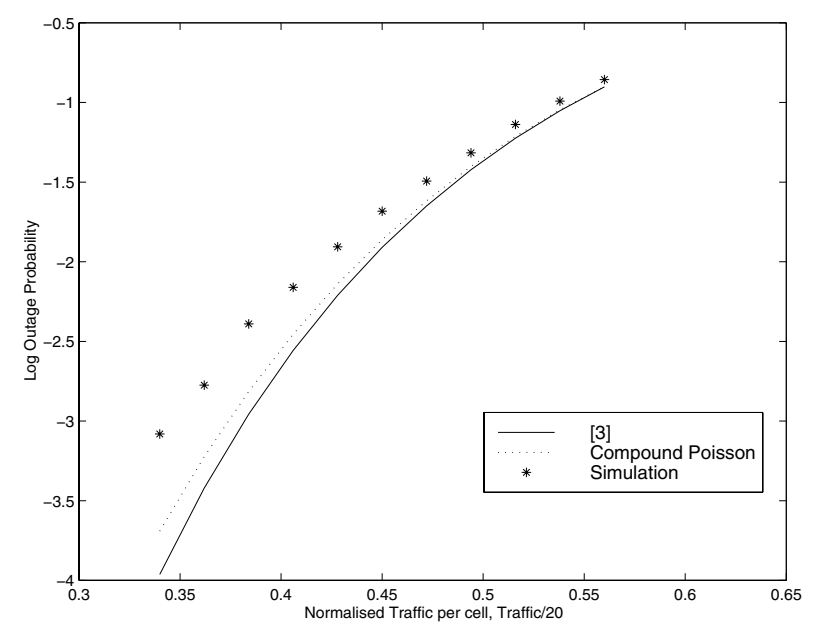

Fig. 3. Comparison of Compound Poisson Method, Approximation in [3] and Simulation for $\Gamma=20$. The user density is uniform.

is

$$
\begin{aligned}
F(x) \sim & P(x)-\left[\frac{\gamma_{1}}{6} Z^{(2)}(x)\right]+\left[\frac{\gamma_{2}}{24} Z^{(3)}(x)+\frac{\gamma_{1}^{2}}{72} Z^{(5)}(x)\right]- \\
& {\left[\frac{\gamma_{3}}{120} Z^{(4)}(x)+\frac{\gamma_{1} \gamma_{2}}{144} Z^{(6)}(x)+\frac{\gamma_{1}^{3}}{1296} Z^{(8)}(x)\right]+} \\
& {\left[\frac{\gamma_{4}}{720} Z^{(5)}(x)+\frac{\gamma_{2}^{2}}{1152} Z^{(7)}(x)+\frac{\gamma_{1} \gamma_{3}}{720} Z^{(7)}(x)+\right.} \\
& \left.\frac{\gamma_{1}^{2} \gamma_{2}}{1728} Z^{(9)}(x)+\frac{\gamma_{1}^{4}}{31104} Z^{(11)}(x)\right]+\ldots
\end{aligned}
$$

$Z^{(i)}(x)$ is the $i^{\text {th }}$ derivative of the Gaussian density with zero mean and unit variance for $Z(x)$ as defined above.

$$
\gamma_{r-2}=\frac{1}{n^{\frac{n}{2}-1}} \frac{\left(\frac{1}{n} \sum_{i=1}^{n} \kappa_{r, i}\right)}{\left(\frac{1}{n} \sum_{i=1}^{n} \sigma_{i}^{2}\right)^{r / 2}}
$$

$\kappa_{r, i}$ as defined above is the $r_{t h}$ cumulant of the $\operatorname{rv} Y_{i}$

Terms in brackets are terms of the same order with respect to $n$. When the $Y_{i}$ have the same distribution, then $m_{i}=m, \sigma_{i}^{2}=\sigma^{2}$, 
$\kappa_{r, i}=\kappa_{r}, \mu_{2, i}=\mu_{2}, \mu_{3, i}=\mu_{3}, \mu_{4, i}=\mu_{4}$

$$
\gamma_{r-2}=\frac{1}{n^{\frac{r}{2}-1}}\left(\frac{\kappa_{r}}{\sigma^{r}}\right)
$$

The terms of order $n^{-\frac{\nu}{2}}$ in Edgeworth's Expansion contain the moments $\mu_{3}, \ldots, \mu_{\nu+2}$, which are precisely the moments required for approximation to this order.

We will denote the truncated Edgeworth series $F_{(i)}(x)$ to be the approximation to $F(x)$, containing only $i$ terms of the expansion in Eqn. (9), other than the CLT term $P(x)$.

For a proof of the Edgeworth Expansion see [8].

\section{D.1 Application to CLT Approximation}

We needed the outage probability given by

$$
P_{\text {out }}=\sum_{n=0}^{\infty} \operatorname{Pr}\left(\sum_{j=1}^{n} I_{j} \geq \Gamma / N=n\right)\left(\frac{e^{-M A}(M A)^{n}}{n !}\right)
$$

Instead of approximating the probability expression directly by applying CLT as,

$$
\operatorname{Pr}\left(\sum_{j=1}^{n} I_{j} \geq \Gamma / N\right) \approx Q\left(\frac{\Gamma-\mu_{n}}{\sigma_{n}}\right)
$$

we approximate using the Edgeworth-Correction as,

$$
\operatorname{Pr}\left(\sum_{j=1}^{n} I_{j} \geq \Gamma / N\right) \approx F_{(2)}\left(\frac{\Gamma-\mu_{n}}{\sigma_{n}}\right)
$$

where we will take only the first two terms of the expansion for correction (using upto the fourth moments of the rv), so

$$
F_{(2)}(x)=[Q(x)+\text { First-Term }- \text { Second-Term }]
$$

The derivation of First-Term (contains upto third moments of y) and Second-Term (contains upto fourth moments of y) of the Edgeworth-Correction is shown in Appendix -A.

The corrected outage probability expression is,

$$
P_{\text {out }} \approx \sum_{n=0}^{\infty} F_{(2)}\left(\frac{\Gamma-\mu_{n}}{\sigma_{n}}\right)\left(\frac{e^{-M A}(M A)^{n}}{n !}\right)
$$

\section{D.2 Results}

We compare the results by applying the Edgeworth Correction to the Compound Poisson Eqn. (11) and the simulations in Fig. 4 and Fig. 5, for $\Gamma=100$ and $\Gamma=20$ respectively. Results show that there is a significant improvement after using the Edgeworth corrections for $P_{\text {out }}$ and it closely approximates the simulation results. As an example in Figure $4(\Gamma=100)$, we see that for an outage probablity of .001, improvement using two terms of Edgeworth Expansion over using CLT is $80 \%$ and the improvement using compound poisson approach over using CLT is $20 \%$. Corresponding values for $\Gamma=20$ are $88 \%$ and $22 \%$.

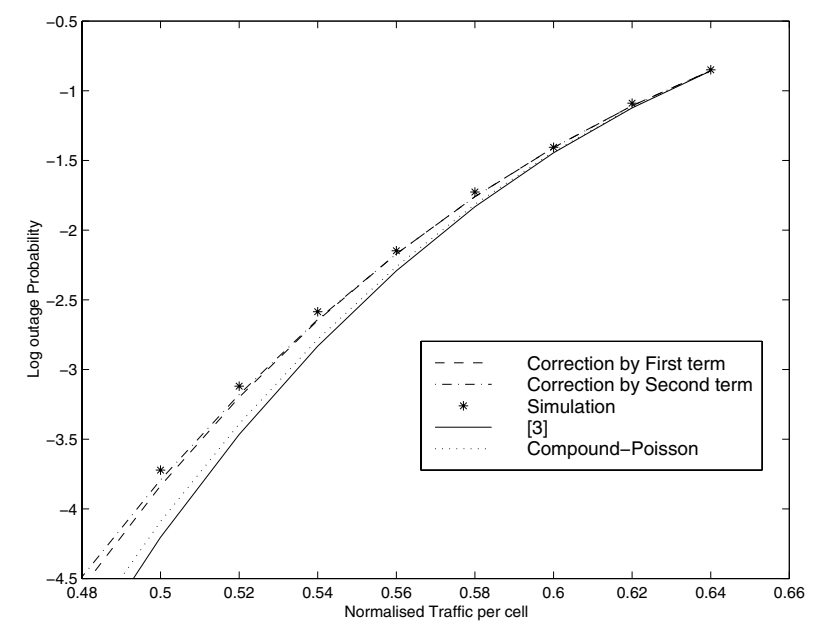

Fig. 4. Edgeworth Correction with first and second terms compared to simulation and [3] for $\Gamma=100$. The user density is uniform.

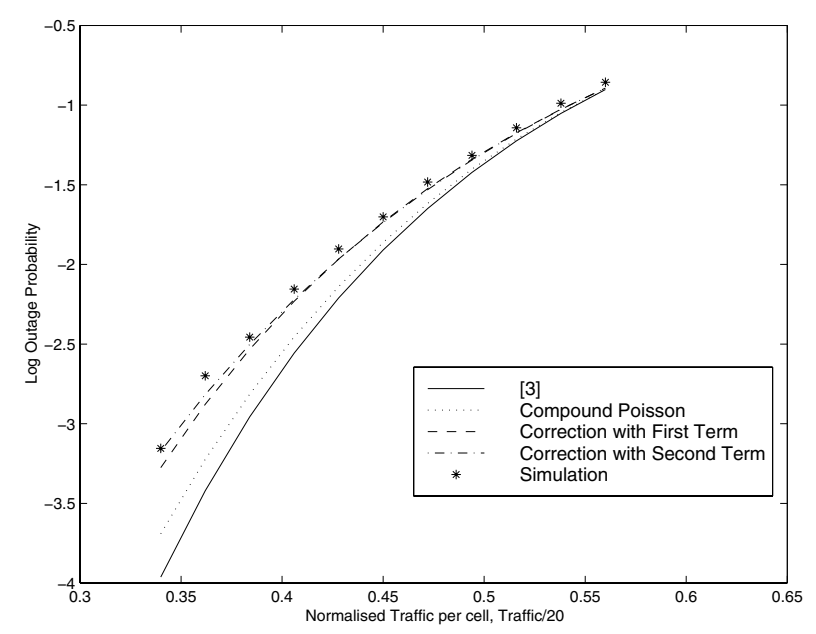

Fig. 5. Edgeworth correction with first and second terms compared to simulation and [3] for $\Gamma=20$. The user density is uniform.

\section{E. Extension to Non-Uniform User Density and Irregular Cells}

Our results are easily extendible to the non-uniform user density case. To do that we will take a specific case of non-uniform density. We assume the user density to be Normalized Truncated Gaussian in the 2-D hexagonal tiling as in Figure 1. For finding the moments of $I$, the individual interference rvs, we use (5) and assume the density of users to be,

$$
f(x, y)=\left[\frac{\frac{\exp \left[\frac{-\left(x^{2}+y^{2}\right)}{2 \sigma^{2}}\right]}{2 \pi \sigma^{2}}}{\iint_{\mathcal{R}} \frac{\exp \left[\frac{-\left(x^{2}+y^{2}\right)}{2 \sigma^{2}}\right]}{2 \pi \sigma^{2}}}\right]
$$

where $x, y \in \mathcal{R}$. Similarly the results are easily extendible to irregular cells by performing the numerical integrations over the irregular region instead of the hexagonal region in the system. We have compared the results obtained by taking the user density to be Normalized Truncated Gaussian in Fig. 6 and 7 for $\Gamma=100$ and $\Gamma=20$ respectively. We can see that the results are quite accurate in this case too. Corresponding values of improvement as in Section(IV-D.2) are $60 \%$ and $20 \%$ for $\Gamma=100$ 


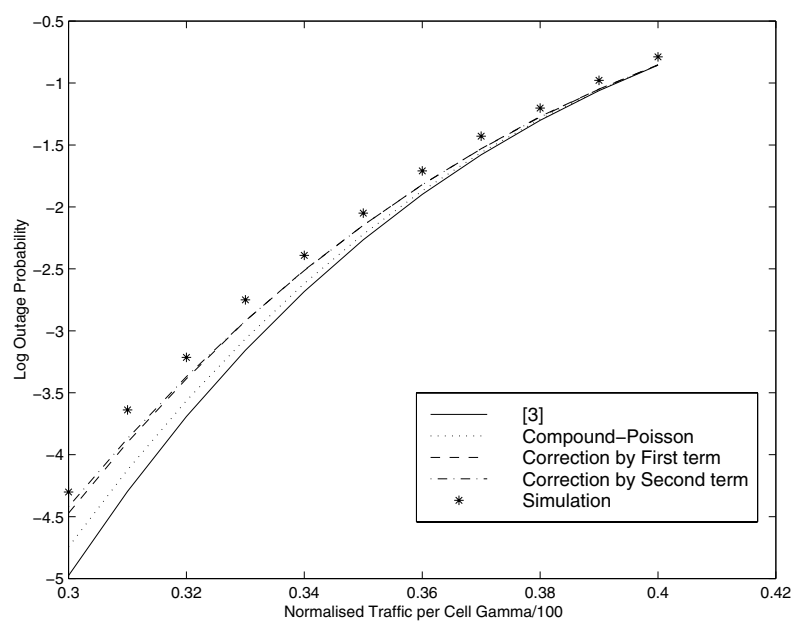

Fig. 6. Edgeworth correction with first and second terms compared to simulation and CLT for $\Gamma=100$. The user density is Normalized Truncated Gaussian.

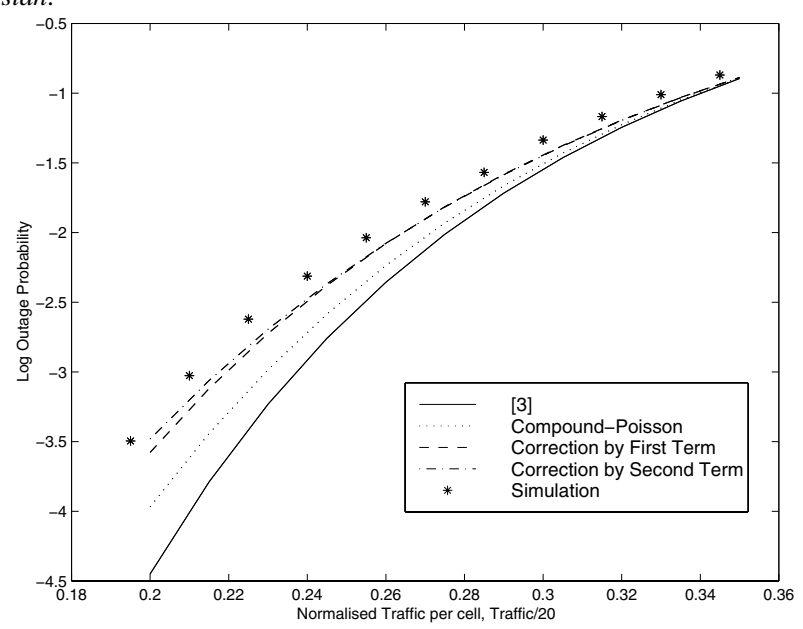

Fig. 7. Comparison of Edgeworth corrections, Compound Poisson, [3] with Simulation for $\Gamma=20$. The user density is Normalized Truncated Gaussian.

and $70 \%$ and $30 \%$ for $\Gamma=20$.

\section{UPPER Bounds FOR the OUtAge PROBABILITy}

In this section we calculate an upper bound for the outage probability. We calculate the large deviation bound using a very effective way, which can then be extended easily to the case of non-uniform user traffic and irregular cells in the system.

\section{A. Chernoff Bound}

We give here an upper bound for the outage probability using the Chernoff bound. From Cramer's theorem applied to i.i.d rvs $x_{i}, i=1,2, \ldots n$, for $a>E(x)$,

$$
\frac{1}{n} \log \operatorname{Pr}\left(\sum_{i=1}^{n} x_{i} \geq n a\right) \leq-l(a)
$$

Where,

$$
l(a)=\sup _{\theta}\left[\theta a-\mu_{x}(\theta)\right]
$$

$\theta$ is real and

$$
\mu_{x}(\theta)=\log E\left[e^{\theta x}\right]
$$

is the log moment generating function (LMGF) of the rv $x$. The above bound is commonly called the Chernoff bound. Applying the above to our problem with $I_{k}=x_{i}$

$$
\frac{1}{n} \log \operatorname{Pr}\left(\sum_{k=1}^{n} I_{k} \geq \Gamma\right) \leq-l(\Gamma / n)
$$

Let

$$
S_{n}=\sum_{k=1}^{n} I_{k}
$$

Then

$$
\operatorname{Pr}\left(S_{n} \geq \Gamma\right) \leq e^{-n l(\Gamma / n)}
$$

where

$$
\begin{gathered}
l(\Gamma / n)=\sup _{\theta}\left[\theta a-\mu_{I}(\theta)\right], \\
\mu_{I}(\theta)=\log E\left[e^{\theta I}\right]
\end{gathered}
$$

and $I$ is the individual interference rv.

\section{B. Calculation of the Bound}

\section{B.1 Compound Poisson Chernoff Bound}

We need to find a bound for

$$
P_{\text {out }}=\sum_{n=0}^{\infty} \operatorname{Pr}\left(\sum_{k=1}^{n} I_{k} \geq \Gamma\right)\left(\frac{e^{-A} A^{n}}{n !}\right)
$$

If each term in the summation of (15) is replaced by its upper bound, then we get an upper bound for $P_{\text {out }}$, since each term in the summation is a product of probabilities and hence positive. This implies,

$$
\begin{aligned}
P_{U B}=\sum_{n=0}^{\infty} P_{u b_{n}}\left(\frac{e^{-A} A^{n}}{n !}\right) \geq & \sum_{n=0}^{\infty} \operatorname{Pr}\left(\sum_{k=1}^{n} I_{k} \geq \Gamma\right) \\
& \left(\frac{e^{-A} A^{n}}{n !}\right)
\end{aligned}
$$

where,

$$
P_{u b_{n}} \geq \operatorname{Pr}\left(\sum_{k=1}^{n} I_{k} \geq \Gamma\right)
$$

Then using Eqn. (14),

$$
P_{u b_{n}}=e^{-n l(\Gamma / n)}
$$

where,

$$
l(\Gamma / n)=\sup _{\theta}\left[\theta \frac{\Gamma}{n}-\mu_{I}(\theta)\right]
$$

and,

$$
\mu_{I}(\theta)=\log E\left[e^{\theta I}\right]
$$

Then to calculate $P_{U B}$, we need to solve an optimization problem,

$$
\max _{\theta} f(\theta)=\left[\frac{\theta \Gamma}{n}-\mu_{I}(\theta)\right]
$$

for each $n$ in the summation of Eqn. (16).

For the calculation of Log Moment Generating Function(LMGF), $\mu_{I}(\theta)=E\left[e^{\theta I}\right]$, we have two methods as discussed in Section V-B.3. 


\section{B.2 Deterministic Sum Approach}

This method is from [3], where the authors replace the compound Poisson sum by a deterministic sum in distribution,

$$
S_{N}=\sum_{k=1}^{N} I_{k} \doteq \sum_{j=1}^{A} Y_{j}
$$

where $Y_{1}, Y_{2}, \ldots Y_{A}$ are iid rvs and

$$
\begin{gathered}
Y_{1} \doteq \sum_{k=1}^{N_{1}} I_{k} \\
E\left[Y_{1}\right]=\mu_{Y}=\mu_{I} \\
\operatorname{Var}\left(Y_{1}\right)=\sigma_{Y}^{2}=\sigma_{I}^{2}+\mu_{I}^{2} . \\
\mu_{Y}(\theta)=e^{\mu_{I}(\theta)}-1
\end{gathered}
$$

Using Eqn. (14) they obtain Chernoff bound from,

$$
\operatorname{Pr}\left(S_{N} \geq \Gamma\right) \leq e^{-A l(\Gamma / A)}
$$

where

$$
\begin{aligned}
l(\Gamma / A) & =\sup _{\theta}\left[\theta \frac{\Gamma}{A}-\mu_{Y}(\theta)\right] \\
& =\sup _{\theta}\left[\theta \frac{\Gamma}{A}+1-e^{\mu_{I}(\theta)}\right]
\end{aligned}
$$

\section{B.3 Calculation of LMGF}

We discuss here two methods to evaluate the LMGF, $\mu_{I}(\theta)$, which is required for the solution of the optimization problems in Eqn. (17) and Eqn. (19).

1. Using Density of Individual Interference RVs [3]: This approach is given by [3], where the authors approximate the hexagonal cells by circles and find the distribution of the individual interference rv depending on the position of the mobile in the system. User density is assumed to be uniform in the system. They obtain a very complicated but closed form expression for the distribution $F_{I}(z)$ of the the interference rvs. We can evaluate the LMGF, $\mu_{I}(\theta)$, using the following Riemann Stieljes Integral,

$$
\mu_{I}(\theta)=E\left[e^{\theta I}\right]=\int_{z} \operatorname{Exp}[\theta z] d F_{I}(z)
$$

The results obtained by this method are not accurate due to the approximations. Moreover in the case of non-uniform user density the evaluation of the distribution will be very difficult.

2. Numerical Integration: This approach is similar to finding the moments of interference. We need to calculate $\mu_{I}(\theta)=$ $E\left[e^{\theta I}\right]$, and for this we evaluate the numerical integral,

$$
E\left[e^{\theta I}\right]=\iint_{\mathcal{R}} \operatorname{Exp}[\theta I(x, y)] f(x, y) d A(x, y)
$$

where $\mathcal{R}$ is the 2-D Hexagonal Tiling as in Fig. 1.

It can be seen that the method is very accurate and we do not need to evaluate the density of individual interference rvs. Moreover it can be very easily extended to the case of nonuniform user density and irregular cells as seen in the Section VC.

\section{Extension to Non-Uniform User Density and Irregular Cells}

For this case we again assume the user density to be Normalized Truncated Gaussian in the 2-D hexagonal tiling as in Fig. 1. The numerical integrations for finding $E\left[\exp ^{\theta I}\right]$ are the same as in Eqn. (21) where the density is given by (12). Similarly the results are easily extendible to systems with irregular cells, by integrating over the irregular cell region. However we are not giving here any numerical examples for that case.

\section{Results}

We compared the Chernoff bounds obtained by using Compound Poisson approach (17) and the deterministic sum approach (19) (both using numerical integrations as in (21)) with the result obtained by simulation in Fig. 8. It was observed that both methods provided almost similar results but the former takes 6 to $7 \mathrm{hrs}$ of computer time, while the latter takes hardly $3 \mathrm{~min}$. So we decided to work with the later approach (deterministic sum approach).

We then compared the Chernoff bounds obtained by using density of individual interference rvs Eqn. (20) and that obtained by numerical integration Eqn. (21), with the simulations, for $\Gamma=100$, in Fig. 9. It is clearly visible that, due to the exactness of numerical integration method, the Chernoff bound obtained is tighter. The time taken by the numerical integration and density methods is almost the same $(3 \mathrm{~min})$. So we decided to work with the later approach (Numerical integration).

Later the results obtained by Chernoff bound (obtained using Eqn. (19) and Eqn. (21) and Edgeworth corrections are compared with simulations, for the uniform user density, in Fig. 10 and 11, and for nonuniform user density case in Fig. 12 and 13, both for $\Gamma=100$ and $\Gamma=20$, respectively.

It can be observed from the above results that the Chernoff upper bound is very conservative.

\section{LOGNORMAL SHADOWING}

In this section we discuss the characterization of interference from other users and approximating the outage probability when lognormal shadowing is included in the propagation model. We look at extending the capacity analyses that includes the effects of shadowing and power control.

We compare the methods of characterizing interference by [1] and [9] and finally we propose to find the moments through Simulation. We will use the propagation model of Eqn. (3) with both distance and shadowing variations in the intereference. We again assume that all the effects of fast Raleigh fading are averaged out and are encapsulated in $E_{b} / I_{0}$ requirements. We take into consideration the dependence of the propagation losses at two different base stations from a mobile user as in [9].

\section{A. Calculation of Outage Probability}

\section{A.1 Method of [1]}

Inclusion of lognormal shadowing and power control makes the analysis of other user interference very complex. This is because an MS must be power controlled by a minimum attenuation BS since then the interference to all other BSs in the system would be minimum. The assumptions of [1] lead to a much simplified analysis but it gives only upper bounds on the mean and 


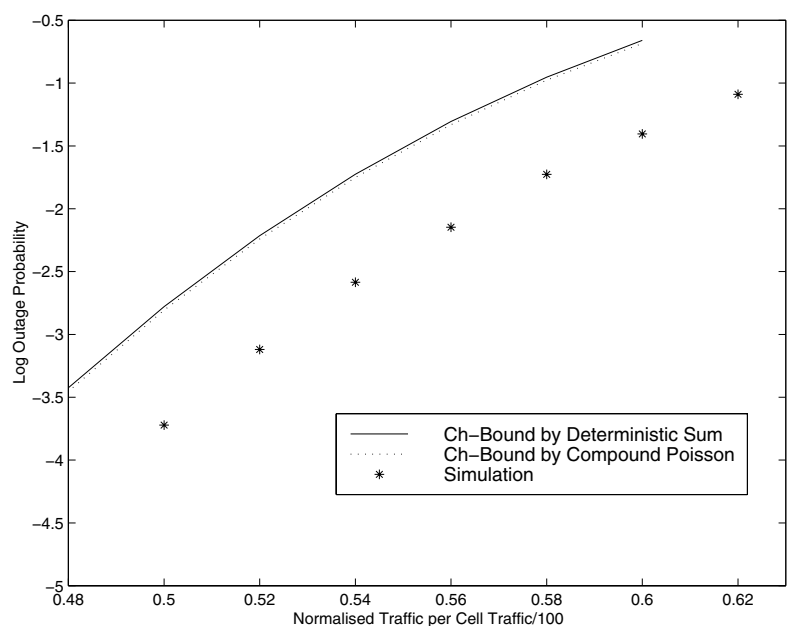

Fig. 8. Comparison of Chernoff Bounds using Compound Poisson and Deterministic Sum ([3]) approaches with Simulation for $\Gamma=100$. The user density is uniform.

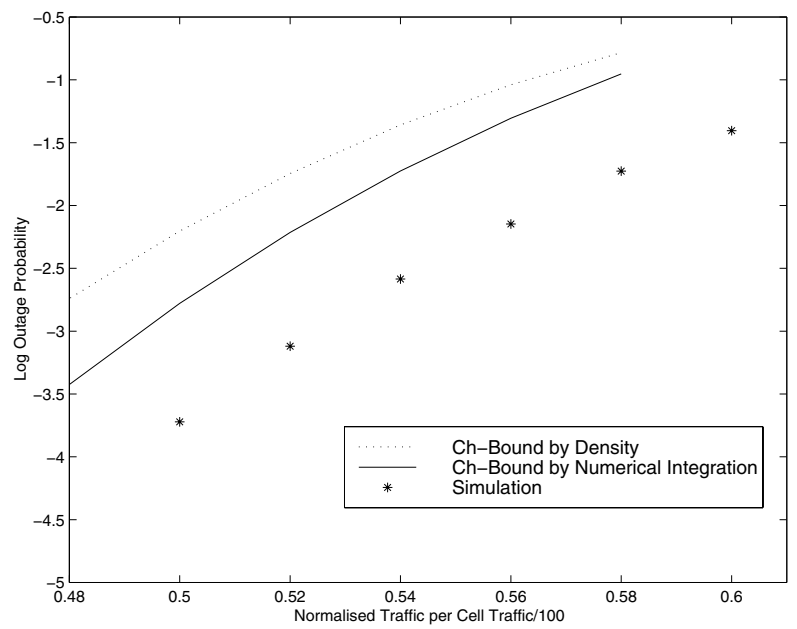

Fig. 9. Comparison of Bounds and Approximations with Simulation for $\Gamma=100$. The user density is uniform.

the second moment calculated through numerical integrations. We applied the method of [1] to calculate the mean and variance of the individual interference rv. In addition to the assumptions in Section 3.1, we also assume here that attenuation in forward and reverse links are the same. This assumption is necessary to allow the MS to choose a BS based only on the signal power received from the BSs. For the expressions of mean and variance and their derivations, refer [1].

We calculated mean and variance and applied the Compound Poisson CLT approximation to obtain outage probability as given in Section IV. This is then compared with the simulations in Figure 14. It can be seen from Figure 14 that the outage probability calculated from Compound Poisson CLT approximation needs Edgeworth corrections in the tail portion. But the authors of [1] do not give any method to calculate the higher moments. Hence this approach is inadequate for accurate capacity calculations. However this is quite a good approximation for the outage probability. We also compare the mean and variance from the Numerical Integration method in [1] the simulation results

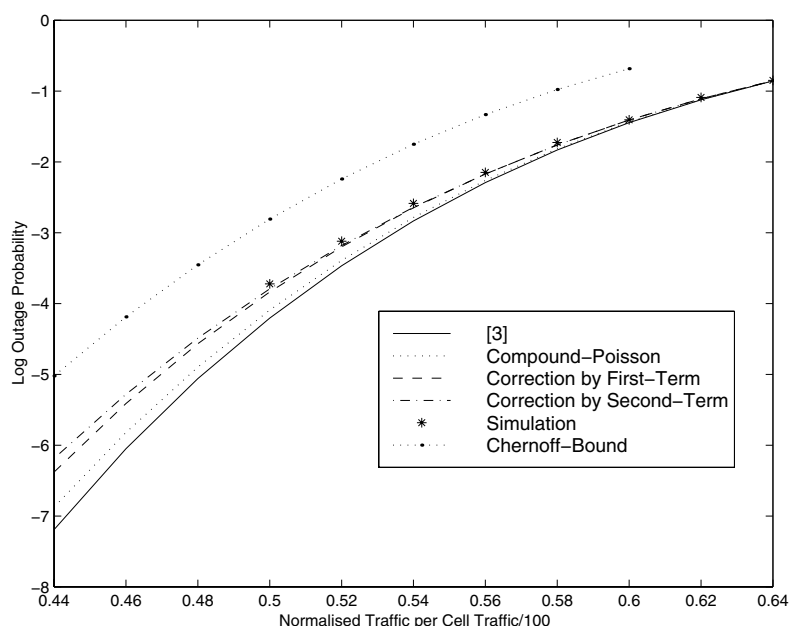

Fig. 10. Comparison of Bounds and Approximations with Simulation for $\Gamma=100$. The user density is uniform.

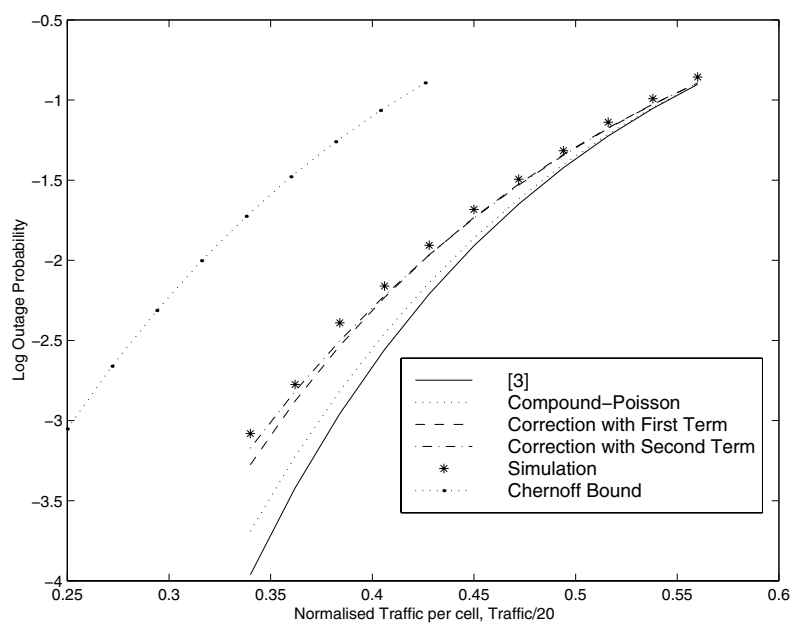

Fig. 11. Comparison of Bounds and Approximations with Simulation for $\Gamma=20$. The user density is uniform.

in Table I.

\section{A. 2 Simulation Method}

Simulation method is same as discussed in Section IV-C.2. But here the method of calculating interference changes. Each MS is assigned a uniform location in the system and its attenuation to all the BSs is computed. An MS is then power controlled by that BS which receives its signal with the least attenuation, i.e., $\left(r_{m}^{4} 10^{\xi_{m} / 10}\right)$, where $r_{m}$ is the distance of a MS from the $m^{t h}$ BS. Then since its transmitted power will be proportional to this minimum attenuation, it will produce the least interference to other cell base stations. In this way the simulations correspond to the case that is likely to be seen in practice. Based on this we then compute the interference to the target BS.

\section{A.3 Method of [9]}

The approach given by [9] is more practical and accurate, but is computationally very complex. Also nothing is said about the second moments. Generalization of the method for calculating the higher moments will become much more complex. 


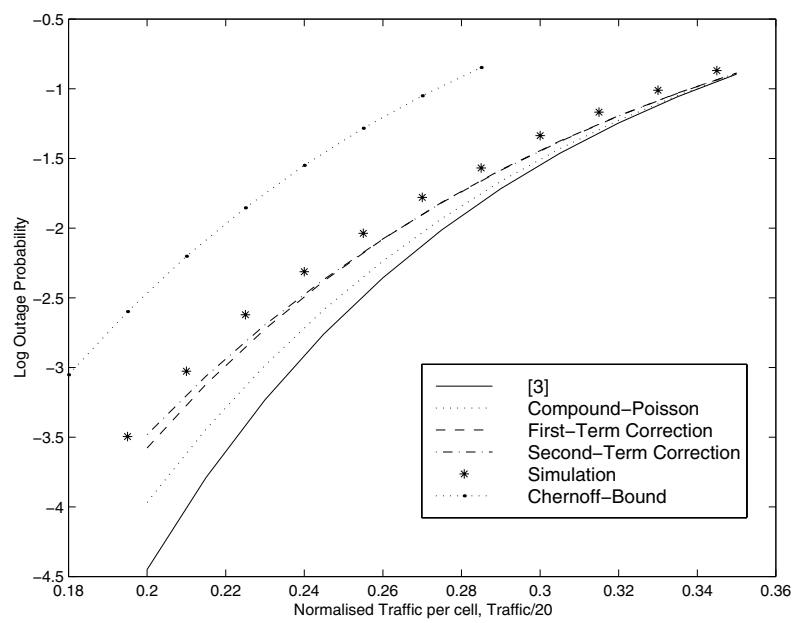

Fig. 12. Comparison of Bounds and Approximations with Simulation, for $\Gamma=100$. The user density is Normalized Truncated Gaussian.

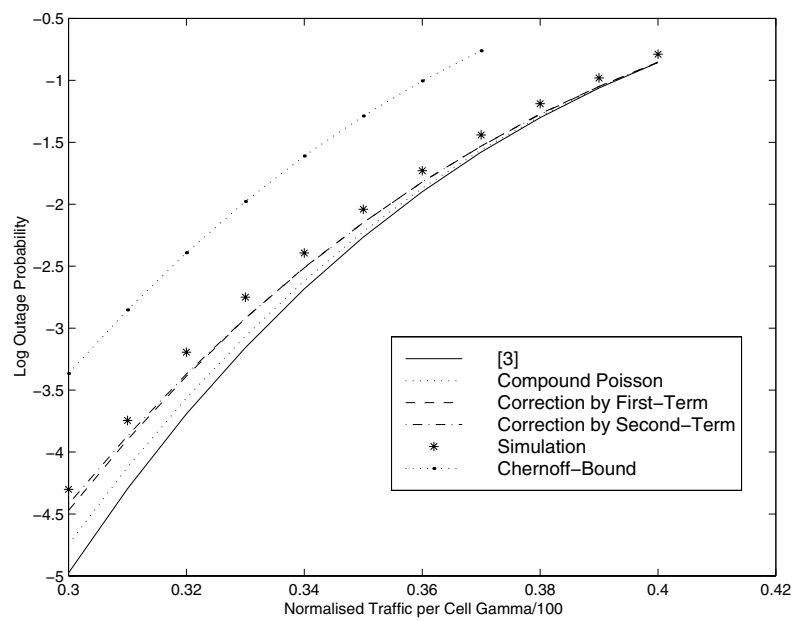

Fig. 13. Comparison of Bounds and Approximations with Simulation, for $\Gamma=20$. The user density is Normalized Truncated Gaussian.

\section{B. Mean and Higher Moments Through the Simulation}

Generally in wireless networks, some of the parameters like standard deviation of lognormal variate $\sigma$, may change with environment, and so they are taken to be empirical estimates. Since the simulation study can allow any amount of complexity to be included, and also because exact analysis for the computation of mean and variance with power control by the leastattenuation-BS is highly complicated and inexact, we propose to estimate the mean, variance and the higher moments to be obtained through simulations. We have compared in Figure 15, the outage probability obtained by CLT approximation and correction by Edgeworth Expansion using mean, variance and higher order moments estimated through simulations, it is observed that almost $98 \%$ correction is obtained for the outage of .001 with two terms of correction by Edgeworth Series. The method of estimating moments through simulation and applying Compound Poisson CLT and Edgeworth corrections, for obtaining outage probability is observed to be computationally much faster than the method for obtaining the outage probability directly through simulations. In the examples considered, the for-

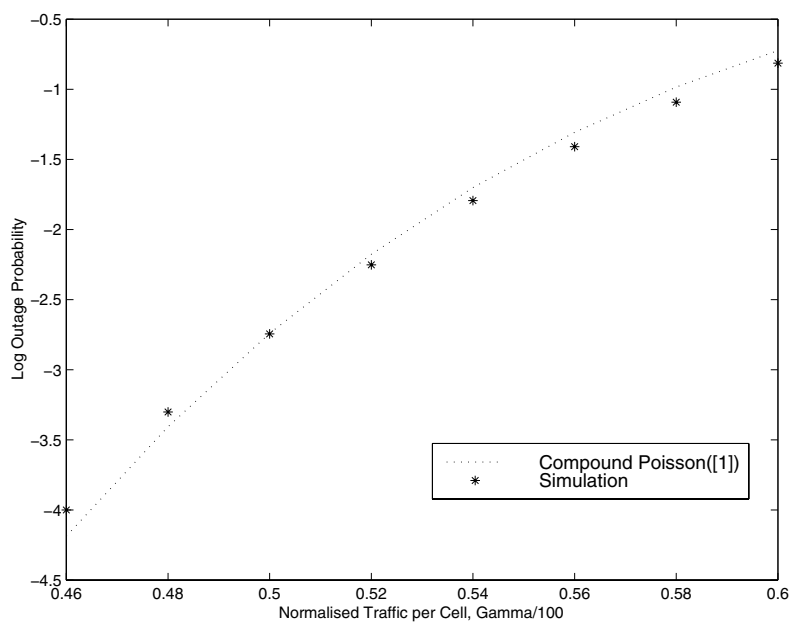

Fig. 14. Outage Probability from the method of [1] Compared to Simulation.

\begin{tabular}{|l||l|l|}
\hline \hline & $\begin{array}{l}\text { Numerical Integration } \\
\text { as in [1]. }\end{array}$ & Simulation \\
\hline \hline Mean & 0.08117 & 0.08004 \\
\hline Variance & 0.05741 & 0.05650 \\
\hline
\end{tabular}

TABLE I

COMPARISON OF MEAN AND VARIANCE OF INDIVIDUAL INTERFERENCE RV OBTAINED THROUGH NUMERICAL INTEGRATION [1] AND THROUGH SIMULATION FOR STANDARD DEVIATION $\sigma=8$, PLE $\gamma=4$.

mer method took 20 minutes, while the latter took us 48 hours on the same computer to obtain the outage probability curve. We have compared the values of the mean of the interference rv obtained from [9] with those obtained from simulations in table II, for validating the estimated mean through the simulations. There is a close agreement in the values of mean, except for the case $N_{c}=1$. There is some inaccuracy inherent in this method for the case $N_{c}=1$, which we could not identify.

\begin{tabular}{|l||l|l|}
\hline \hline & $\begin{array}{l}\text { Numerical Integration } \\
\text { as in [9]. }\end{array}$ & Simulation \\
\hline \hline$N_{c}=1$ & 0.177 & 0.161 \\
\hline$N_{c}=2$ & 0.0932 & 0.0934 \\
\hline$N_{c}=3$ & 0.0826315 & 0.082031 \\
\hline$N_{c}=4$ & 0.0816 & 0.081283 \\
\hline
\end{tabular}

TABLE II

COMPARISON OF MEAN OF INDIVIDUAL INTERFERENCE RV OBTAINED THROUGH NUMERICAL INTEGRATION [9] AND THROUGH SIMULATION FOR STANDARD DEVIATION $\sigma=8$, PLE $\gamma=4$.

\section{CONCLUSION}

Our aim was to develop efficient analytic techniques for capacity calculations of systems employing CDMA. In this last section we briefly reiterate the main results and conclusions of this paper.

We first gave a novel method for characterizing an individual 


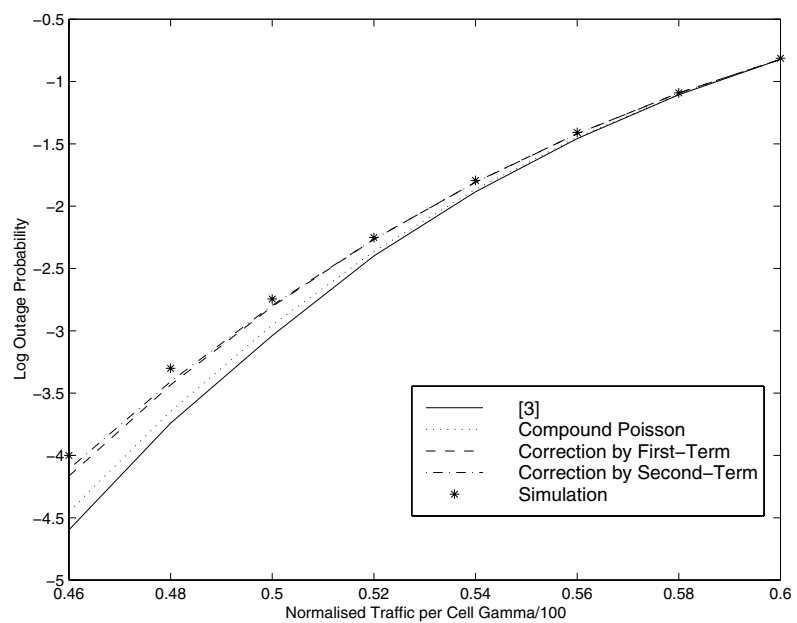

Fig. 15. Edgeworth correction using simulated moments, compared to simulation and Compound Poisson for $\Gamma=100$.

interference random variable in Section IV. For simple propagation models with no shadowing, using numerical integrations over 2-D hexagonal regions it was shown that it is possible to obtain all moments of interference rv. This method was shown to be easily extendible to the case of nonuniform user traffic and irregular cells in the system, to obtain outage probability. To eliminate the error in CLT approximation, Edgeworth asymptotic expansions were used. It was shown with the help of numerical examples that the error is reduced by an order of magnitude and the outage probability closely approximates the simulations. It is necessary to mention here that the error in CLT approximation was such that the resulting outage probability overestimated the capacity, which is a matter of concern. Our method eliminates this error and should be useful for the design of future mobile cellular networks, which are going to operate at very low outage probabilities. Numerical examples for the case of nonuniform user density were also shown.

In Section V we obtained upper bounds for the outage probability. The method for calculation of moments in Section IV was extended to calculate the Log Moment Generating Function.

Section VI, includes lognormal shadowing. Some existing approaches for moment calculation in this case were shown to be inadequate. Later we showed using numerical example that use of simulations to obtain moments, and then applying CLT and Edgewoth corrections, allows us to include any amount of complexity in the system, while estimating the outage probability accurately. Possible future work is:

1. We believe that CLT is not an approximation but a lower bound. However it remains to be proved.

2. There is a need to obtain some analytical method for finding the moments of interference rvs, when lognormal shadowing is included in the propagation model, and MS is power controlled by the least attenuation BS.

\section{APPENDIX}

\section{A. Calculation of the Terms of Edgeworth Expansion}

From Eqn. (9),

$$
\begin{gathered}
\text { Fisrt-Term }=\left[\frac{\gamma_{1}}{6} Z^{(2)}(x)\right] \\
\text { Second-Term }=\left[\frac{\gamma_{2}}{24} Z^{(3)}(x)+\frac{\gamma_{1}^{2}}{72} Z^{(5)}(x)\right]
\end{gathered}
$$

Since we want to approximate the distribution of $\sum_{j=1}^{n} I_{j}$ where $I_{j} s$ are iid rvs, $\gamma_{1}$ and $\gamma_{2}$ can be obtained using Eqn. as

$\gamma_{1}=\frac{1}{\sqrt{n}}\left(\frac{\kappa_{3}}{\sigma^{3}}\right)$

Since, $\kappa_{3}=\mu_{3}=E\left(I^{3}\right)-3 m E\left(I^{2}\right)+2 m^{3}$, we get, $\gamma_{1}=\frac{1}{\sqrt{n}}\left(\frac{\mu_{3}}{\sigma^{3}}\right)$ and $\gamma_{2}=\frac{1}{n}\left(\frac{\kappa_{4}}{\sigma^{4}}\right)$ where $\kappa_{4}=\mu_{4}-$ $3 \mu_{2}^{2}$. In Eqns. (22) and (23), $Z^{(2)}(x), Z^{(3)}(x)$ and $Z^{(5)}(x)$ are the derivatives of the Gaussian density with zero mean and unit variance,

$$
Z(x)=\frac{1}{\sqrt{2 \pi}} e^{-x^{2} / 2}
$$

and,

$$
\begin{gathered}
Z^{(2)}(x)=\exp \left(-x^{2} / 2\right)\left(x^{2}-1\right) \\
Z^{(3)}(x)=\exp \left(-x^{2} / 2\right)\left(-x^{3}+3 x\right) \\
Z^{(5)}(x)=\exp \left(-x^{2} / 2\right)\left(-15 x+10 x^{3}-x^{5}\right)
\end{gathered}
$$

Using all the derivatives, and the moments of individual interference rv calculated using Eqn. (5) we obtain the First-Term and Second-Term of the Edgeworth corrections to the CLT approximation.

\section{REFERENCES}

[1] K. S. Gilhousen, I. M. Jacobs, R. Padovani, A. J. Viterbi, L. A. Weaver and C. E Wheatley, "On the Capacity of a cellular CDMA System,"

[2] A. J. Viterbi, A. M. Viterbi,"Erlang Capacity of a Power Controlled CDMA System," IEEE Journal on Selected Areas in Communications, Vol.11 No. 6, August 1993 pp. 892-900.

[3] Jamie S. Evans and David Everitt, "On the Teletraffic Capacity of CDMA Cellular Networks," IEEE Trans. Veh. Technology, vol.48 no.1, pp. 153165, Jan. 1999.

[4] Leonard Kleinrock, "Queueing Systems," John Wiley and Sons Vol.I, 1975 pp. 234.

[5] William Feller,"An Introduction to Probability Theory and Its Applications,”Wiley Eastern Private Ltd., Vol.1 1970 pp. 288.

[6] Milton Abramowitz and Irene A. Stegun, "Handbook of Mathematical Functions," Dover Publications, Inc., New York, 1970, pp. 935.

[7] Personal correspondence with Jamie S. Evans was useful in considering this method.

[8] Herald Cramér, "Mathematical Methods of Statistics," Princeton University Press, p.213 and p.231, Princeton 1946.

[9] A. J. Viterbi, A. M. Viterbi, K. S. Gilhousen and E.Zehavi,"Soft Handoff Extends CDMA Cell Coverage and Increases reverse Link Capacity," IEEE Journal on Selected Areas in Communications, Vol.12 No. 8, October 1994 pp. 1281-1288.

[10] A. J. Viterbi,"Principles of Spread Spectrum Communication," AddisonWesley Wireless Communications Series. 